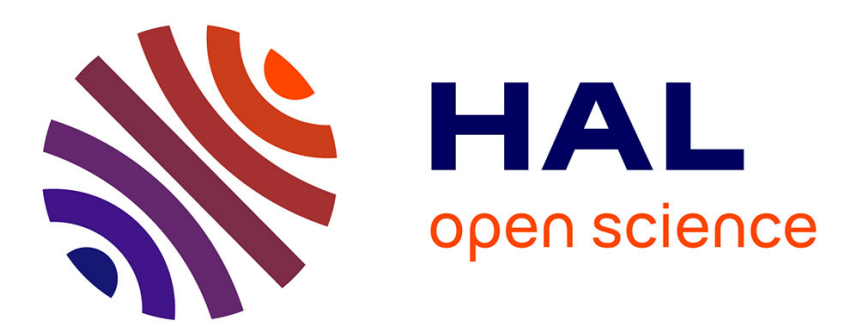

\title{
Modeling of the very low pressure helium flow in the LHC Cryogenic Distribution Line after a quench
}

Benjamin Bradu, Silviu-Iulian Niculescu, Philippe Gayet, Emmanuel Witrant

\section{To cite this version:}

Benjamin Bradu, Silviu-Iulian Niculescu, Philippe Gayet, Emmanuel Witrant. Modeling of the very low pressure helium flow in the LHC Cryogenic Distribution Line after a quench. Cryogenics, 2010, 50, pp.71-77. 10.1016/j.cryogenics.2009.11.004 . hal-00452782

\section{HAL Id: hal-00452782 \\ https://hal-centralesupelec.archives-ouvertes.fr/hal-00452782}

Submitted on 3 Feb 2010

HAL is a multi-disciplinary open access archive for the deposit and dissemination of scientific research documents, whether they are published or not. The documents may come from teaching and research institutions in France or abroad, or from public or private research centers.
L'archive ouverte pluridisciplinaire HAL, est destinée au dépôt et à la diffusion de documents scientifiques de niveau recherche, publiés ou non, émanant des établissements d'enseignement et de recherche français ou étrangers, des laboratoires publics ou privés. 


\title{
Modeling of the very low pressure helium flow in the LHC Cryogenic Distribution Line after a quench
}

\author{
Benjamin Bradu ${ }^{\mathrm{a}, \mathrm{b}}$, Philippe Gayet ${ }^{\mathrm{a},}$, Silviu-Iulian Niculescu ${ }^{\mathrm{b}}$, Emmanuel Witrant $^{\mathrm{c}}$, \\ ${ }^{a}$ CERN, EN Department CH-1211 Genève 23, Switzerland \\ ${ }^{b}$ Laboratoire des Signaux et Systèmes, UMR CNRS 8506, CNRS-Supélec, 3 rue Joliot Curie, 91192, Gif-sur-Yvette, France \\ ${ }^{c}$ Gipsa-lab,University Joseph Fourier, B.P. 46, 38402, ST. Martin d'Héres, France
}

\begin{abstract}
This paper presents a dynamic model of the helium flow in the Cryogenic Distribution Line (QRL) used in the Large Hadron Collider (LHC) at CERN. The study is focused on the return pumping line, which transports gaseous helium at low pressure and temperature $(1.6 \mathrm{kPa} / 3 \mathrm{~K})$ over $3.3 \mathrm{~km}$. Our aim is to propose a new real-time model of the QRL while taking into account the non-homogeneous transport phenomena. The flow model is based on 1D Euler equations and considers convection heat transfers, hydrostatic pressure and friction pressure drops. These equations are discretized using a finite difference method based on an upwind scheme. A specific model for the interconnection cells is also proposed. The corresponding simulation results are compared with experimental measurements of a heat wave along the line that results from a quench of a superconducting magnet. Different hypotheses are presented and the influence of specific parameters is discussed.
\end{abstract}

Key words: Helium (B), Heat transfer (C), Fluid Dynamics (C), Numerical Simulation (F), Transmission lines (F)

\section{Introduction}

In 2008, the European Organization for Nuclear Research (CERN) started the most powerful particle accelerator of the world, the Large Hadron Collider (LHC). The LHC accelerates proton beams which are driven by superconducting magnets maintained at $1.9 \mathrm{~K}$ over a $27 \mathrm{~km}$ ring. To cool-down and maintain superconductivity in different magnets, large helium refrigeration plants are used [1].

The LHC cryogenic systems are divided into 8 equivalent cryoplants around the LHC ring and each of them supply helium to superconducting magnets over $3.3 \mathrm{~km}$ via a cryogenic distribution line (called QRL) installed underground in parallel to magnets [2]. The dynamic behavior of helium flows in the QRL is not well understood, especially during transients as after a quench. To the best of the authors knowledge, relevant dynamic models for such a cryogenic line do not exist, due to the system specificities (length, interconnections, gaseous helium at cryogenic temperature and at low pressure). Thus, this paper provides a numerical dynamic model Bradu)

Email addresses: ben jamin.bradu@cern.ch (Benjamin

Published in Cryogenics 2010; 50:71-77 for gaseous helium flows in long cryogenic lines that contain interconnections with heat transfers.

Previously, a real-time dynamic simulator for cryogenic systems called PROCOS and using the modeling software EcosimPro ${ }^{\odot}$ was developed at CERN [3]. In this context, the model of a $1.8 \mathrm{~K}$ refrigeration unit for the LHC achieved to simulate the pumping over the QRL performed by the cold-compressors [4].

The model of the QRL flow is embedded in a larger model that aims at operator training, diagnostic and control improvements. Thus, the QRL model must be computed fast enough to obtain simulations faster than realtime in case of operator training.

First, the paper presents briefly the cryogenic distribution line of the LHC and its interconnections to the main cooling loops. Then, the numerical model of the return pumping line at very low pressure is detailed: the model of the flow, the heat transfers and the pressure drops are described with their numerical implementations in Section 3. Next, steady-state and dynamic simulations during a quench are presented in Section 4. The presented results are discussed and compared with real data obtained in 2008.

January 31, 2010 


\section{The Cryogenic Distribution Line}

The QRL is composed of five different headers (called B-F) with temperatures ranging between $4 \mathrm{~K}$ and $75 K$ to supply magnets, beam screens and thermal shields of the magnet cryostats, see Figure 1. All main QRL elements as inner headers and internal fixed points are built in stainless steel AISI $304 \mathrm{~L}$.

A LHC sector of $3.3 \mathrm{~km}$ is divided into different cryogenic cells that constitute the elementary cooling loops. A sector is composed of 23 standard cells of $106,9 \mathrm{~m}$ (composed of 6 dipoles and 2 quadripoles) plus other specific cells according to the sector considered, see Figure 2 where the sector 5-6 of the LHC is represented. The main cooling loops are supplied in supercritical helium by the header $\mathrm{C}$ and the helium flows are returned back to the header B through sub-cooled heat exchangers.

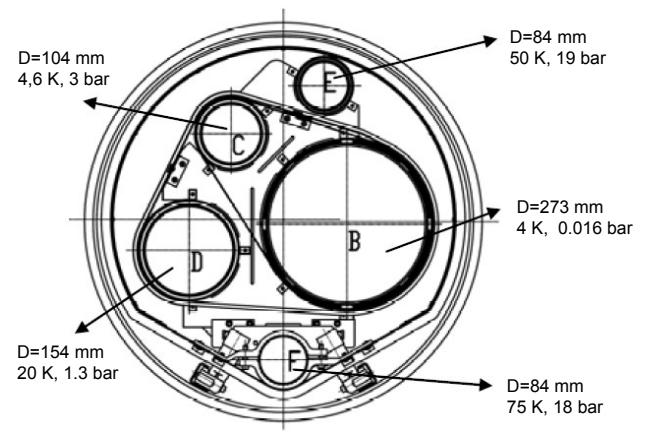

Figure 1: QRL cross-section with remarkable data

\section{Modeling of the header B}

Helium is considered as a perfect fluid : viscosity and thermal conductivity are neglected as helium has a very low viscosity $\left(\mu \approx 10^{-6}\right.$ Pa.s) and a very low thermal conductivity $\left(\lambda \approx 10^{-3} \mathrm{~W} \cdot \mathrm{m}^{-1} \cdot \mathrm{K}^{-1}\right)$. Moreover, gaseous helium is considered as a compressible gas.

\subsection{Modeling of the Flow}

First, the main flow line is modeled, neglecting interconnections. According to previous assumptions, an inviscid flow in the header B is considered. Thus, the flow can be described by Euler equation which is obtained from Navier-Stokes equation neglecting the viscosity and the thermal conductivity [5]. The conservative form of Euler equations is the following:

$$
\frac{\partial}{\partial t}\left[\begin{array}{c}
\rho \\
\vec{M} \\
E
\end{array}\right]+\vec{\nabla} \cdot\left[\begin{array}{c}
\rho \cdot \vec{V} \\
\rho \cdot \vec{V}^{T} \otimes \vec{V}+P \cdot I \\
\rho \cdot \vec{V} \cdot\left(u+\frac{P}{\rho}\right)
\end{array}\right]=\left[\begin{array}{l}
0 \\
0 \\
q
\end{array}\right]
$$

where the different variables are described in Table 1. These three equations represent mass, momentum and energy balances. The momentum is defined as $\vec{M}=$ $\rho \cdot \vec{V}$ and the total energy per unit volume is $E=\rho$. $\left(u+\frac{1}{2} \cdot\left(V_{x}^{2}+V_{y}^{2}+V_{z}^{2}\right)\right)$.

\begin{tabular}{|c|c|c|}
\hline Symbol & Description & Unit \\
\hline$c$ & Sound velocity & $m \cdot s^{-1}$ \\
\hline$D$ & Diameter & $m$ \\
\hline E & Energy per unit volume & $J . m^{-3}$ \\
\hline$f r$ & Darcy-Weisbach friction factor & - \\
\hline$h$ & Specific enthalpy & $J . \mathrm{kg}^{-1}$ \\
\hline$h_{c}$ & Heat transfer coefficient & $W \cdot m^{-2} \cdot K^{-1}$ \\
\hline$k$ & Thermal conductivity & $W \cdot m^{-1} \cdot K^{-1}$ \\
\hline$L$ & Length & $m$ \\
\hline$\dot{m}$ & Mass flow & $k g . s^{-1}$ \\
\hline$\vec{M}$ & Momentum & $k g \cdot m^{-2} \cdot s^{-1}$ \\
\hline$P$ & Pressure & $\mathrm{Pa}$ \\
\hline $\operatorname{Pr}$ & Prandtl number & - \\
\hline$R e$ & Reynolds number & - \\
\hline$S$ & Hydraulic cross section & $m^{2}$ \\
\hline$q$ & External heat inputs & $W \cdot m^{-3}$ \\
\hline$T$ & Temperature & $K$ \\
\hline$u$ & Specific internal energy & $J . \mathrm{kg}^{-1}$ \\
\hline$\vec{V}$ & Gas speed & $m \cdot s^{-1}$ \\
\hline$\mu$ & Viscosity & Pa.s \\
\hline$\rho$ & Density & kg. $m^{-3}$ \\
\hline$g=9.81$ & Gravity acceleration & $m \cdot s^{-2}$ \\
\hline$R=8.31$ & Perfect Gaz constant & J.mol ${ }^{-1} \cdot K^{-1}$ \\
\hline$R_{H e}=2078$ & Helium specific constant & $J . \mathrm{kg}^{-1} \cdot K^{-1}$ \\
\hline$C_{v}=3148$ & Helium specific heat & $J . \mathrm{kg}^{-1} \cdot \mathrm{K}^{-1}$ \\
\hline$\gamma=1.66$ & Specific heat ratio, $\frac{C_{p}}{C_{v}}$ & - \\
\hline
\end{tabular}

The following assumptions on the flow are assumed :

- flux according to the $x$ direction only (the main flow direction) $: V=V_{x}$ and $M=\rho \cdot V_{x}$;

- straight line. The QRL curvature (radius of curvature of $4.3 \mathrm{~km}$ ) has a negligible impact on the flow;

- in operational conditions, the kinetic component can be neglected: $\rho \cdot|\vec{V}|^{2}<<$, which implies that $\rho \cdot \vec{V}^{T} \otimes \vec{V}+P \cdot I \approx P \cdot I$.

Considering the above approximations, Euler equation (1) can be expressed in 1D as:

$$
\frac{\partial X(x, t)}{\partial t}+F(X) \cdot \frac{\partial X(x, t)}{\partial x}=Q(x, t)
$$




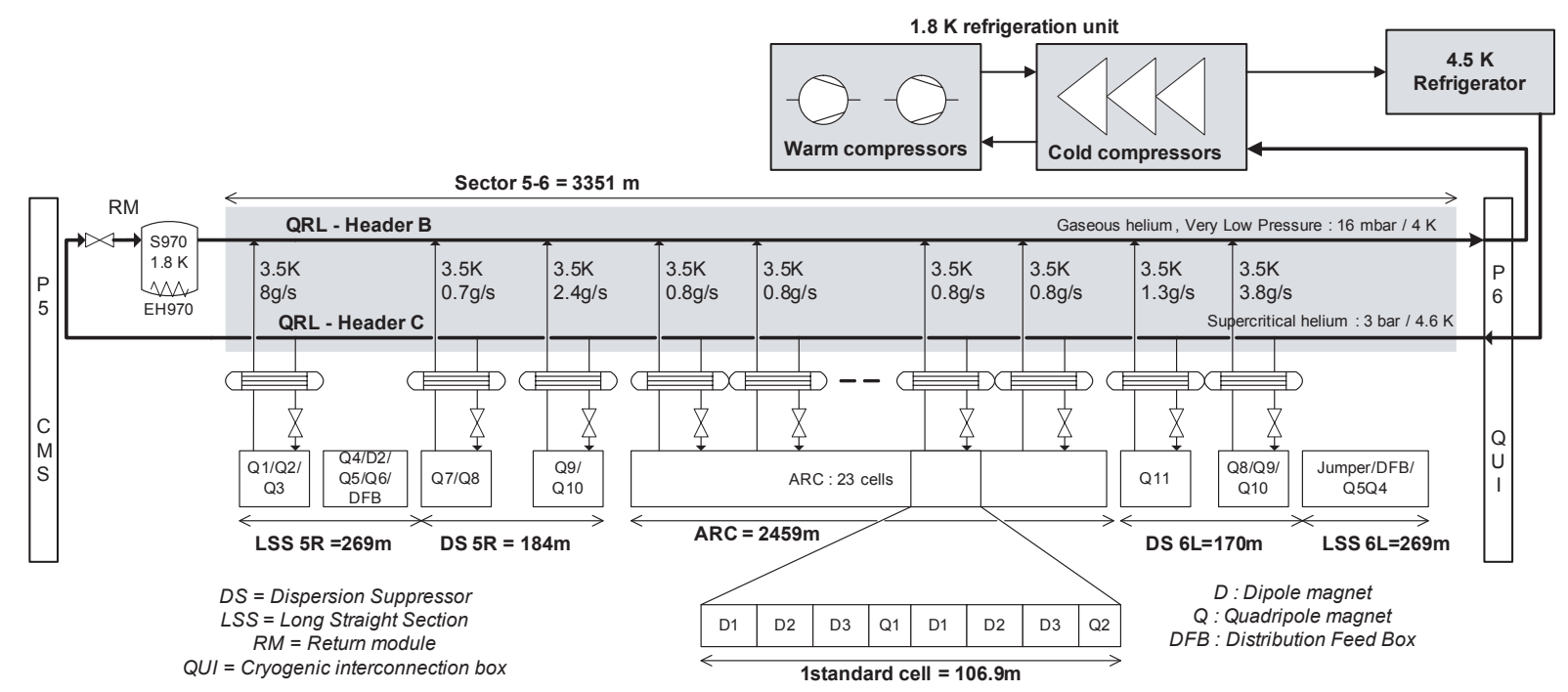

Figure 2: Details of the LHC sector 5-6 with the main cooling loops for the superconducting magnets

where $X=\left[\begin{array}{lll}\rho & M & E\end{array}\right]^{T}$ is the state vector, $F$ is the Jacobian flux matrix and $Q=\left[\begin{array}{lll}0 & 0 & q\end{array}\right]^{T}$ is the source vector.

\subsubsection{Modeling for an ideal gas flow}

To compute the Jacobian flux matrix, it is necessary to include an equation of state to link pressure, density and internal energy. First, an ideal gas is considered: it is assumed that $u=C_{v} \cdot T$, which leads to the following equation of state:

$$
P=\rho \cdot R \cdot T=\rho \cdot u \cdot \frac{R}{C_{v}}=\rho \cdot u \cdot \hat{\gamma}
$$

where the constant $\hat{\gamma}=(\gamma-1)$. For this case, the Jacobian matrix was calculated in $[5,6]$ as:

$$
F=\left[\begin{array}{ccc}
0 & 1 & 0 \\
\frac{(\gamma-3) V^{2}}{2} & (3-\gamma) V & \hat{\gamma} \\
\hat{\gamma} V^{3}-\frac{\gamma V E}{\rho} & \frac{\gamma E}{\rho}-\frac{3 \hat{\gamma} V^{2}}{2} & \gamma V
\end{array}\right]
$$

and the eigenvalues of the Jacobian are:

$$
\left\{\begin{array}{l}
\lambda_{1}=V+c \\
\lambda_{2}=V \\
\lambda_{3}=V-c
\end{array}\right.
$$

where $c$ is the speed of sound, defined by:

$$
c=\sqrt{\gamma R T}=\sqrt{\frac{\gamma P}{\rho}}=\sqrt{\gamma \hat{\gamma}\left(\frac{E}{\rho}-\frac{V^{2}}{2}\right)}
$$

All the eigenvalues of the Jacobian are real and distinct. This means that Eq. (2) is a strictly hyperbolic system of equations. Moreover, for subsonic flows
$(V<c)$ there are two positive eigenvalues and one negative eigenvalue: informations are propagated forward and backward and the eigenvalues represent the different speeds of propagation.

\subsubsection{Modeling for a gaseous helium flow}

The previous equations cannot be directly applied to helium flows at low temperature as the equation of state (3) is not valid anymore. Hence, the following empirical formulation for the helium internal energy is considered:

$$
u=u_{0}+C_{v} \cdot T
$$

where $u_{0}=14950 \mathrm{~J} \mathrm{~kg}^{-1}$ is a constant. This equation remains valid for gaseous helium at low pressure $(P<$ $10 \mathrm{kPa}$ ), with a relative error less than $1 \%$ between $1.8 \mathrm{~K}$ and $300 \mathrm{~K}$. For higher pressures, this equation is still valid for gaseous helium far from the saturation line and far from the critical point.

We are interested in simulating the helium flow during the final cool-down of magnets from $4.5 \mathrm{~K}$ to $1.8 \mathrm{~K}$. During this phase, the pressure in the header $\mathrm{B}$ decreases from $0.1 \mathrm{MPa}$ to $1.6 \mathrm{kPa}$ and temperatures are between $2 K$ and $10 K$. Within these ranges, Eq. (7) remains valid and the equation of state and the sound velocity for helium become respectively:

$$
\begin{aligned}
& P=\rho \cdot R_{H e} \cdot T=\rho \cdot\left(u-u_{0}\right) \cdot \hat{\gamma} \\
& c=\sqrt{\gamma \hat{\gamma}\left(\frac{E}{\rho}-\frac{V^{2}}{2}-u_{0}\right)}
\end{aligned}
$$


A new Jacobian flux matrix is computed with additional terms containing the constant $u_{0}$ :

$$
F=\left[\begin{array}{ccc}
0 & 1 & 0 \\
\frac{(\gamma-3) V^{2}}{2}-u_{0} \hat{\gamma} & (3-\gamma) V & \hat{\gamma} \\
\hat{\gamma} V^{3}-\frac{\gamma V E}{\rho} & \frac{\gamma E}{\rho}-\hat{\gamma}\left(\frac{3 V^{2}}{2}+u_{0}\right) & \gamma V
\end{array}\right](10)
$$

and the eigenvalues are the same as for an ideal gas, see Eq. (5). Thus, the system of equations is still a strictly hyperbolic system with the same propagation speeds than for ideal gases but the dynamics is different.

\subsection{Discretization Scheme}

To solve numerically the system of partial differential equations, the flow is discretized in $N$ nodes, see Figure 3. A finite difference method was chosen to compute space and time derivatives using a first order upwind scheme, as suggested in [6]. The time discretization is performed according to an implicit discretization scheme based on a backward Euler method. As the cryogenic line is pumped by cold-compressors, natural Dirichlet boundary conditions are set as follows:

- the input energy $E(0, t)=E_{1}(t)$;

- the input density $\rho(0, t)=\rho_{1}(t)$;

- the output momentum $M(L, t)=M_{N}(t)$.

Hence, mass and energy are propagated forward whereas momentum is propagated backward, which is in agreement with the signs of eigenvalues obtained in Eq. (5) and with the physics of the flow. As the coldcompressors are 'pulling' helium atoms in the line, the momentum propagation is backward whereas the mass (helium atoms) and energy (heat) are naturally transported with the flow. Thus, in the framework of the upwind scheme, $\partial \rho / \partial x$ and $\partial E / \partial x$ are approximated by a first-order backward finite difference and $\partial M / \partial x$ is approximated by a first-order forward finite difference. The following algebraic system is then obtained:

$$
\begin{array}{r}
\dot{X}_{i}(t)+\frac{A_{i}\left(X_{i}\right)}{\Delta x} X_{i}(t)+\frac{B_{i}\left(X_{i}\right)}{\Delta x} X_{i-1}(t) \\
+\frac{C_{i}\left(X_{i}\right)}{\Delta x} X_{i+1}(t)=Q_{i}(t)
\end{array}
$$

where $i$ denotes the value at $x_{i}$ and:

$$
X_{i}=\left[\begin{array}{c}
\rho_{i} \\
M_{i} \\
E_{i}
\end{array}\right]
$$

$$
\begin{aligned}
& A_{i}=\left[\begin{array}{ccc}
0 & 1 & 0 \\
-\frac{(\gamma-3) V_{i}^{2}}{2}+u_{0} \hat{\gamma} & -(3-\gamma) V_{i} & -\hat{\gamma} \\
\hat{\gamma} V_{i}^{3}-\frac{\gamma V_{i} E_{i}}{\rho_{i}} & \frac{\gamma E_{i}}{\rho_{i}}-\hat{\gamma}\left(\frac{3 V_{i}^{2}}{2}+u_{0}\right) & \gamma V_{i}
\end{array}\right] \\
& B_{i}=\left[\begin{array}{ccc}
0 & -1 & 0 \\
0 & 0 & 0 \\
-\hat{\gamma} V_{i}^{3}+\frac{\gamma V_{i} E_{i}}{\rho_{i}} & -\frac{\gamma E}{\rho_{i}}+\hat{\gamma}\left(\frac{3 V_{i}^{2}}{2}+u_{0}\right) & \gamma V_{i}
\end{array}\right] \\
& C_{i}=\left[\begin{array}{ccc}
0 & 0 & 0 \\
\frac{(\gamma-3) V_{i}^{2}}{2}-u_{0} \hat{\gamma} & (3-\gamma) V_{i} & \hat{\gamma} \\
0 & 0 & 0
\end{array}\right] \\
& Q_{i}=\left[\begin{array}{c}
0 \\
0 \\
q_{i}
\end{array}\right]
\end{aligned}
$$

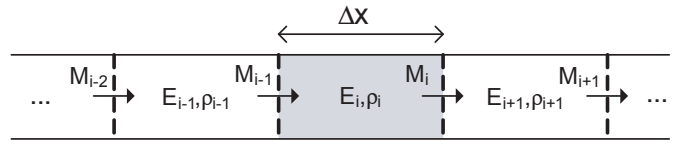

Figure 3: Discretization of the QRL main flow

\subsection{Modeling of interconnections}

Additional helium fluxes coming from LHC magnets enter in the header B every $106.9 \mathrm{~m}$ in standard cells as depicted in Figure 2. These additional fluxes are perpendicular to the main flux but their contribution to the momentum is considered as additive, since it is small compared to the total flow.

Interconnections are included in the discretization scheme at regular intervals every $N_{s u b}$ nodes. Thus, for $N_{\text {inter }}$ interconnections, the line is discretized in $N_{\text {sub }} \cdot N_{\text {inter }}$ nodes, see Figure 4.

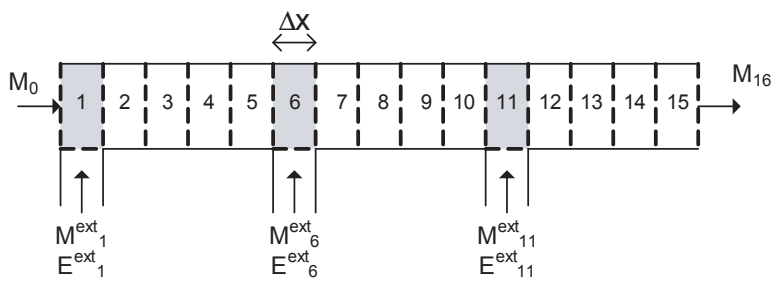

Figure 4: Discretization of the QRL with interconnections for $N_{\text {inter }}=$ 3 and $N_{\text {sub }}=5$

The source term $Q_{i}$ of interconnection nodes is now augmented to include the incoming mass, momentum 
and energy, and consequently writes as:

$$
Q_{i}=\left[\begin{array}{c}
\frac{M_{i}^{\text {ext }}}{\Delta x} \\
(3-\gamma) V_{i}^{\text {ext }} \frac{M_{i}^{\text {ext }}}{\Delta x} \\
q_{i}+\frac{\gamma E_{i}^{\text {ext }} M_{i}^{\text {ext }}}{\rho_{i}^{\text {ext }} \cdot \Delta x}-(\gamma-1)\left(\frac{3 V_{i}^{\text {ext }}}{2}+u_{0}\right) \frac{M_{i}^{\text {ext }}}{\Delta x}
\end{array}\right] \text { (12) }
$$

for $i=(j-1) \cdot N_{\text {sub }}+1$ with $j=1 \ldots N_{\text {inter }}$. Superscripts ext refer to external inputs, namely the input fluxes at interconnections. As LHC magnet cooling circuits are not simulated, the input fluxes variables are the boundary conditions, determined from mass-flow, pressure and temperature measurements on the real plant.

\subsection{Heat transfers}

The term $q_{i}$ in the energy balance represents heat transfers between the fluid and its environments per unit volume in a node. The QRL is well insulated inside a vacuum jacket using a Multi Layer Insulation (MLI) and thermal shields to minimise radiation and conduction losses.

Heat losses can be divided in two parts: static losses $q_{\text {stati }}$ due to radiation, conduction and vacuum barriers and dynamic losses $q_{c o n v i}$ due to convection between the stainless steel pipe and the fluid:

$$
q_{i}=q_{\text {stati }}+q_{\text {convi }}\left(t, \rho_{i}, M_{i}, E_{i}\right)
$$

In a former CERN study, the static heat losses were evaluated at $1.92 \mathrm{~W} / \mathrm{m}^{3}$ in the header $\mathrm{B}$, this corresponds to $0.1 \mathrm{~W} / \mathrm{m}$ along the line [7].

To compute dynamically the convection heat transfer between the pipe and the fluid, supplementary variables are introduced to characterize the pipe properties: its temperature $T_{w}$, its specific heat $C_{p w}$, its mass $M_{w}$ and its internal surface $S_{w}$. The headers are built in stainless steel 304L and the specific heat can be computed from an empirical logarithmic polynomial of the $8^{\text {th }}$ order valid between $2 K$ and $300 K$ [8]. The convective heat transfer coefficient $h_{c}$ is computed by:

$$
h_{c i}=\frac{N u_{i} \cdot k_{i}}{D}
$$

where the Nusselt number $\mathrm{Nu}$ is computed from the Colburn formulation [9]:

$$
N u_{i}=0.023 \cdot \operatorname{Pr}_{i}^{1 / 3} \cdot \operatorname{Re}_{i}^{0.8}
$$

because the flow regime in the header B of the QRL is always turbulent (Reynolds number $R e>10^{5}$ ). Finally the convection heat transfer per unit volume is computed by the Newton law:

$$
q_{c o n v i}=\frac{h_{c i} \cdot S_{w i} \cdot\left(T_{w i}-T_{i}\right)}{S \cdot \Delta x}=\frac{M_{w i}}{S \cdot \Delta x} \cdot C_{p w i} \cdot \frac{d T_{w i}}{d t}(16)
$$

\subsection{Pressure drops}

The QRL is not perfectly horizontal, the slope varies between $-1.5 \%$ and $+1.5 \%$ along the LHC ring, and it results a hydrostatic pressure. This hydrostatic pressure is not taken into account in previous equations and should be considered.

Moreover, friction phenomena are not embedded (the flow is considered inviscid) whereas frictions can lead to pressure drops not negligible in the case of a very low pressure flow as in the QRL. Thus, we propose to replace the momentum partial differential equation $(\partial M / \partial t+\partial P / \partial x=0)$ by an algebraic equation to compute the mass-flow $\dot{m}_{i}$ in each cell (compartmental approach).

The pressure in a cell is computed using the equation of state (8) and the total pressure drop in a cell can be computed as the sum of the hydrostatic pressure difference and the friction pressure drop :

$$
\Delta P_{i}=P_{i}-P_{i+1}=\rho_{i} \cdot g \cdot d z_{i}+f r_{i} \cdot \frac{\Delta x}{D} \cdot \frac{\dot{m}_{i}^{2}}{2 \cdot \rho_{i} \cdot S^{2}}(17)
$$

where $d z_{i}$ is the elevation over the cell $i$ and $f r_{i}$ is the Darcy-Weisbach friction factor. The line B of the QRL can be considered as a smooth pipe and the flow is always turbulent with a Reynolds number $R e>2 \cdot 10^{4}$. In this case, the friction factor can be computed from the empirical formulation of Kakac, Shah and Aung [12] :

$$
f r_{i}=0.184 \cdot R e_{i}^{-0.2}=0.184 \cdot\left(\frac{D \cdot \dot{m}_{i}}{S \cdot \mu_{i}}\right)^{-0.2}
$$

Combining (17) and (18) the mass flow is calculated as function of the total pressure drop:

$$
\dot{m}_{i}=\left(\frac{\left(P_{i}-P_{i+1}\right)-\rho_{i} \cdot g \cdot d z_{i}}{0.184 \cdot\left(\frac{D}{S \cdot \mu_{i}}\right)^{-0.2} \cdot \frac{\Delta x}{2 \cdot D \cdot \rho_{i} \cdot S^{2}}}\right)^{1 / 1.8}
$$

Finally, the momentum is simply deduced from $\dot{m}_{i}$ adding the interconnection momentum :

$$
M_{i}=\frac{\dot{m}_{i}}{S}+M_{i}^{e x t}
$$

\subsection{Numerical implementation}

The modeling and simulations are performed on a commercial software called EcosimPro ${ }^{\complement}$, which is able to simulate systems of differential and algebraic equations (DAEs) [10]. Ecosimpro ${ }^{\complement}$ uses a DASSL algorithm to solve the DAE system [11]. This method is based on an implicit time discretization scheme:

$$
\dot{y}(t)=\frac{y_{n}-y_{n-1}}{t_{n}-t_{n-1}}
$$


The non-linear system dynamics is then solved by iterations for time $t_{n}$ using an implicit Newton-Raphson method. The iteration matrix required by NewtonRaphsons method calculates a Jacobian matrix numerically using finite differences. Note that the use of an implicit scheme renders the time integration robust with respect to numerical errors but its main drawback is the associated lack of precision.

\section{Results and discussion}

\subsection{Simulation in steady-state}

The first task consists in checking that the steadystate reached by the model under constant boundary conditions agrees with the measured values on the QRL. The header B is equipped with temperature sensors every $200 m$ but there is only one pressure sensor and one mass-flow sensor at the end of the header. Each interconnection also has a virtual flow meter allowing to estimate the mass-flow in each cell and the electrical heater power in the phase separator S970 allows us to estimate the mass-flow at the entrance of the header B.

Each of the 8 LHC sectors have the same configuration but they present some differences at their boundaries. A lot of experimental data were collected in one of these sectors during the hardware commissioning in 2008, namely sector 5-6 (chosen for this study). Constant boundary conditions were set in the model, in agreement with the values measured in the sector 5-6 during April and May 2008, when the sector was cold in steady-state. Pressure and temperature at the entrance were set respectively at $1.630 \mathrm{kPa}$ and $1.8 \mathrm{~K}$, the output mass-flow was set to $58 \mathrm{~g} / \mathrm{s}$ and the different external input fluxes are listed in the Table 2.

Static heat losses were evaluated theoretically at $1.92 \mathrm{~W} / \mathrm{m}^{3}$ on the header B but after measurements on the real line, those losses were reevaluated at $1.1 \mathrm{~W} / \mathrm{m}^{3}$, which represent a loss of $0.07 \mathrm{~W} / \mathrm{m}$ along the line.

Table 2: Boundary conditions for input fluxes in sector 5-6

\begin{tabular}{cccc}
\hline Location & Description & $\dot{m}(g / s)$ & $T(K)$ \\
\hline LSS-5R & Q1/Q2/Q3 & 8 & 3.5 \\
DS-5R & Q7/Q8 & 0.7 & 3.5 \\
DS-5R & Q9/Q10 & 2.4 & 3.5 \\
ARC & 23 cells & 0.8 & 3.5 \\
DS-6L & Q11 & 1.3 & 3.5 \\
DS-6L & Q8/Q9/Q10 & 3.8 & 3.5 \\
\hline
\end{tabular}

Figure 5 shows the simulated temperatures, pressures and mass-flows along the line obtained in steady-state (curves) in comparison with sensor values (crosses).
The simulated temperatures agree with the observed ones except for the temperature located at $700 \mathrm{~m}$, due to a poor sensor calibration or to a bad approximation of the first external fluxes. The pressure drop calculated including the hydrostatic pressure and friction pressure drops gives a total pressure drop of $110 \mathrm{~Pa}$ over the $3.3 \mathrm{~km}$. This pressure drop is pretty low in the case of the sector 5-6 because it has a negative slope of $-1.54 \%$ and hydrostatic pressure compensates friction pressure drops.
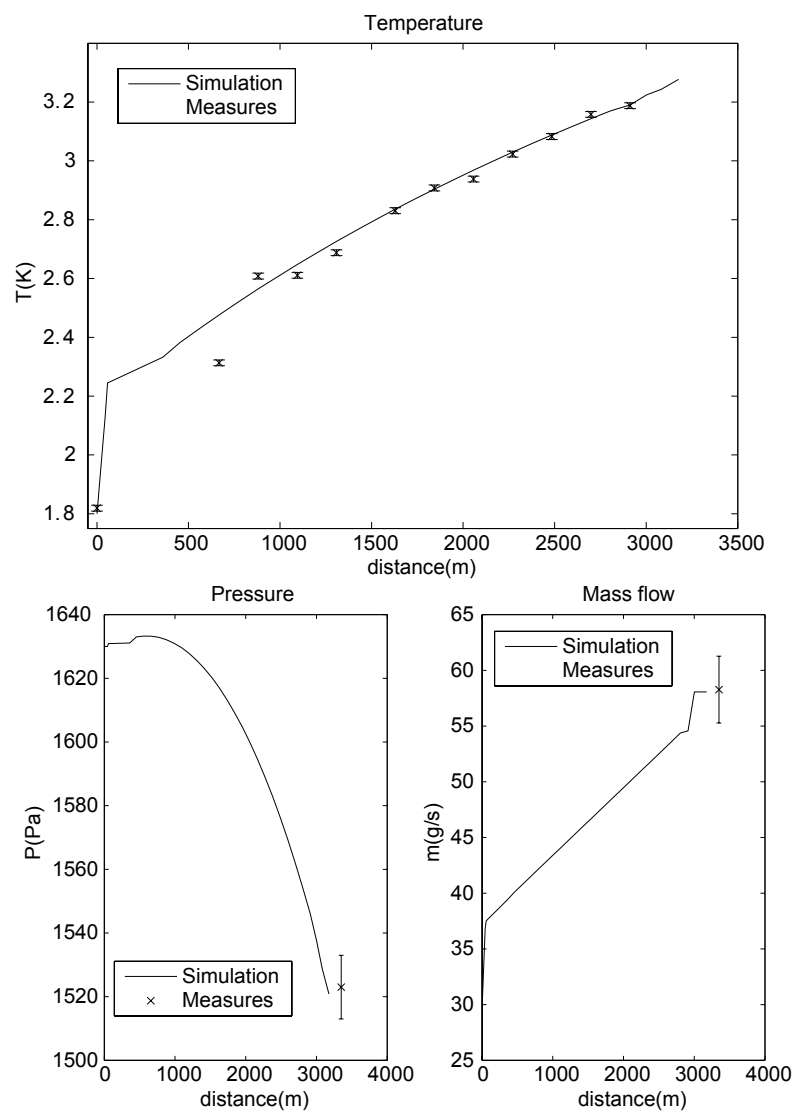

Figure 5: Temperatures, pressures and mass-flows along the header B in steady-state compared with experimental measurements

\subsection{Dynamic simulation during a quench}

When a quench occurs in a superconducting magnet, the resistive transition releases a huge amount of energy. A quench protection system was designed to discharge this energy in large electrical resistances in case of a quench, but an important heat flux is briefly induced in the headers B and D of the QRL (the return lines), thus creating a propagated heat wave. 
In April and May 2008, 23 quenchs were provoked in sector 5-6 during the hardware commissioning of the LHC. It was chosen to reproduce a quench provoked on a dipole magnet located $774 \mathrm{~m}$ after the beginning of header $\mathrm{B}$, inducing a heat wave on more than $2 \mathrm{~km}$.

The quench is simulated by setting a peak at the interconnection boundary (external momentum and temperature) corresponding to the quench location. Due to the lack of instrumentation on the external fluxes, the momentum and temperature peaks are set in such a way that the temperature peak observed on the line B after 107 meters has the correct amplitude and shape. The temperature and mass flow coming from the flux at the quench location are approximated by a bell shape with a peak of $30 \mathrm{~K}$ and $2.6 \mathrm{~g} / \mathrm{s}$ (the temperature of $30 \mathrm{~K}$ is approximatively the temperature reached by the magnet after the quench).

The first simulation is performed with $N=31$ nodes of $106.9 \mathrm{~m}$ each (one node per cooling loop), the convective heat transfer and the pressure drops are neglected $\left(h_{c}=0\right.$ and $\left.d P=0\right)$. Therefore, $\Delta x=106.9 \mathrm{~m}$ and a time step $\Delta t=1 \mathrm{~s}$ is chosen (no special condition is required as an implicit discretization scheme is used). The simulation with these parameters shows that the heat wave is much more dissipated (smaller amplitude and larger width) and much faster in simulation, see Figure 6, where simulation results are plotted in comparison with experimental measurements made on the real line after a quench at different locations.

Then, the convection heat transfer is included in the model. The simulation result is more in agreement with the measurements, as the heat wave is slowed down by the convection transfers. Nevertheless, the heat peak is still too much dissipated.

In order to reduce spatial and time dissipation, the number of nodes is increased by a factor $10(N=310)$. Therefore, the spatial step is reduced to $\Delta x=10.69 \mathrm{~m}$. This simulation shows that the dissipation of the heat peak was reduced and that the simulation result is close to the experimental measurements, but the wave is still a little bit faster in simulation. Note that there are no significant modifications when there are more than 310 nodes.

A fourth simulation was performed including hydrostatic pressure and friction pressure drops using the algebraic equation (19) instead of the partial differential equation to compute the momenta. The result obtained is very similar to the previous one but the heat wave is a little bit slowed and the peak is slightly reduced. This configuration is more in agreement with the measurements and we can consider this simulation as satisfying.

The temperature rise observed on measurements after the quench that is not observed in simulations mainly comes from the approximation of the external flux coming from the quench location.

Simulations are performed on a classical computer, with a processor Pentium ${ }^{\circledR}$ D $3.4 \mathrm{GHz}$ and $1 \mathrm{~GB}$ of RAM. The heat wave generated by the quench is propagated over 20 minutes and for $N=31$, the simulation is 3.4 times faster than real time (6 $\mathrm{min})$, whereas for $N=310$ the simulation takes $8 \mathrm{~min}$. Moreover, the use of the algebraic equation including pressure drops instead of the partial differential equation to compute the momentum increases the simulation speed by a factor 1.5 .

\section{Conclusion}

In this paper, a very low pressure gaseous helium flow model based on 1D Euler equation was used to describe the flow dynamics and heat transfers in a header of the LHC cryogenic distribution line over $3.3 \mathrm{~km}$. Comparisons between real data obtained on the LHC and simulations after a quench have shown that the model is good enough to predict the dynamical behavior of the flow over the line.

These simulations highlight the importance to include the convection heat transfer between the fluid and the pipe in the model to obtain a correct propagation speed and to reproduce a good attenuation of the heat wave. In addition, pressure variations along the line due to hydrostatic pressure and friction pressure drops can be embedded in the model using an algebraic equation to compute momenta instead of the classical partial differential equation. These pressure variations can be significant to simulate the flow dynamics in long cryogenic line presenting a slope.

Moreover, it was shown that the numerical scheme directly impacts the results and deserves a dedicated analysis. The spatial step has to be small enough because the discretization induces dissipation phenomena.

This model can be applied to other gaseous helium flow in long cryogenic distribution lines if helium is far enough from the saturation line, where the equation of state (8) remains valid. For instance, this model works in the headers D, E and F of the QRL but not in the header $\mathrm{C}$, where helium is in the supercritical state, just above the helium critical point.

\section{References}

[1] Lebrun $\mathrm{Ph}$. Cryogenics for the Large Hadron Collider. IEEE Trans App Superc 1999;10:1500-1506. 

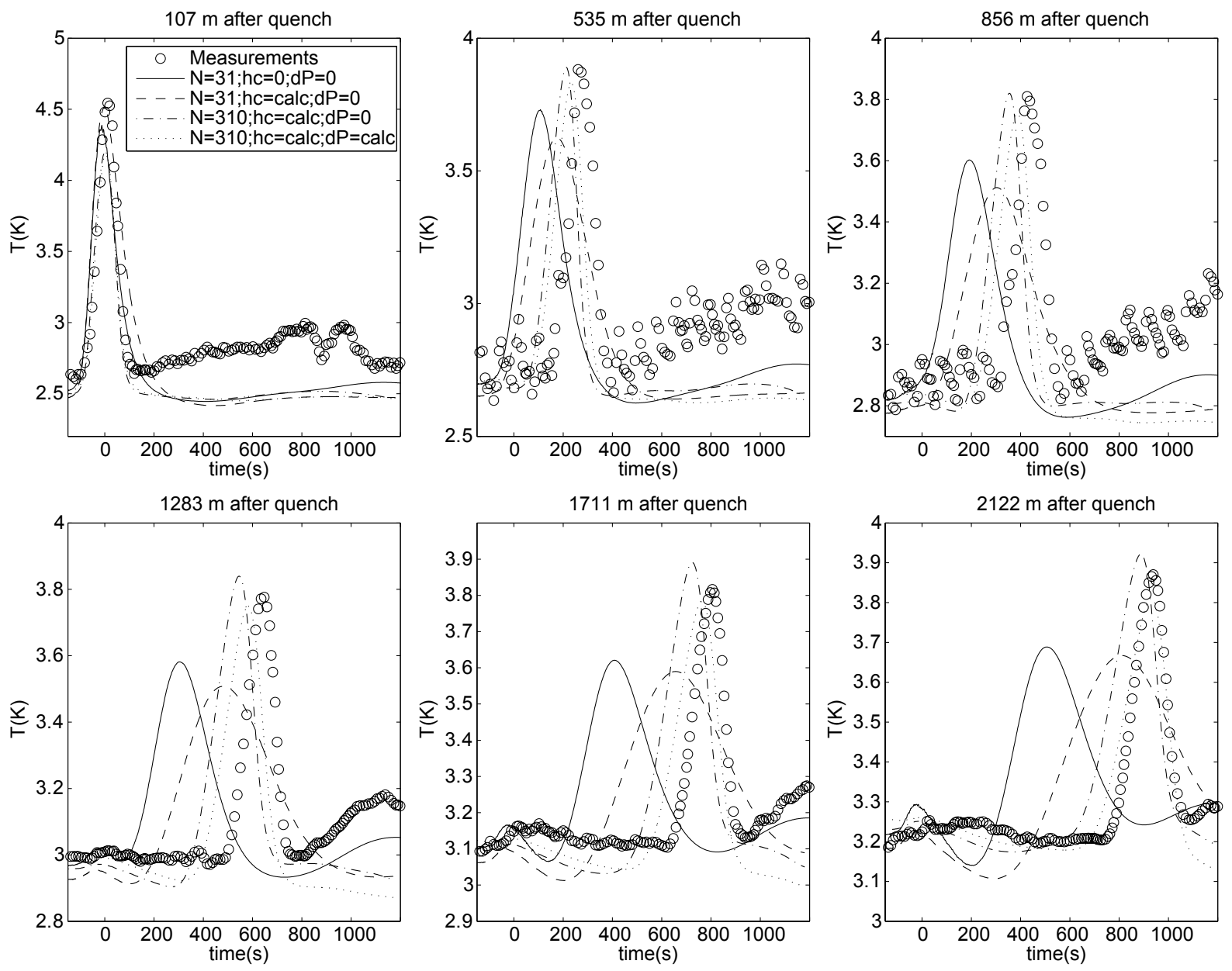

Figure 6: Temperatures along the header B after a quench (real measurements compared to 4 simulations)

[2] Erdt W., Riddone G., Trant R. The cryogenic distribution line for the LHC : functional specification and conceptual design. Adv. Cryog. Eng. 2000;45(B):1387-1394.

[3] Bradu B., Gayet P., and Niculescu, S.I. A process and control simulator for large scale cryogenic plants, Control Engineering Practice 2009, 17:1388-1397.

[4] Bradu B., Gayet P., Niculescu, S.I. Dynamic Simulation of a 1.8 K Refrigeration Unit for the LHC. Proceedings of the 22nd International Cryogenic Engineering Conference, July 2008.

[5] Hirsch C. Numerical Computation of Internal and External Flow, Vol. 2, Chapter 3. John Wiley and Sons, 1990.

[6] Toro E.F. Riemann Solvers and Numerical Methods for Fluid Dynamics, second edition. Springer 1999.

[7] Riddone G. Cryogenic distribution line heat loads. CERN Heat Load Working Group Minutes number 5. 2000.

[8] Marquardt E.D., Le J.P., Radebaugh R. Cryogenic Material Properties Database. Proceedings of the 11th International Cryocooler Conference, June 2000. p.681-687.

[9] Colburn A.P. A method of correlating forced convection heat transfer Data and a comparison with fluid friction, Trans. AIChE 1933; 19:174-210.

[10] EA International. Ecosimpro mathematical algorithms and simulation guide, 2007.
[11] Petzold L.R. A description of DASSL: A Differential/Algebraic System Solver. Sandia National Laboratories Report SAND828637, 1984.

[12] Kakac S.R., Shah K. and Aung W. Handbook of Single-Phase Convective Heat Transfer. John Wiley and Sons 1987. 